\title{
Atomic Data for Stellar Nucleosynthesis
}

\author{
Christopher Sneden $^{1}$, James E. Lawler ${ }^{2}$, Elizabeth A. Den Hartog ${ }^{2}$ \\ and Michael E. Wood ${ }^{2}$ \\ ${ }^{1}$ Department of Astronomy and McDonald Observatory, The University of Texas, \\ Austin, TX 78712 \\ email: chris@verdi.as.utexas.edu \\ ${ }^{2}$ Department of Physics, University of Wisconsin-Madison, 1150 University Ave., \\ Madison, WI 53706 \\ email: jelawler@wisc.edu, eadenhar@wisc.edu, michael.wood@nist.gov
}

\begin{abstract}
Stellar chemical composition analyses can only yield reliable abundances if the atomic transition parameters are accurately determined. During the last couple of decades a renewed emphasis on laboratory spectroscopy has produced large sets of useful atomic transition probabilities for species of interest to stellar spectroscopists. In many cases the transition data are of such high quality that they play little part in the abundance uncertainties. We summarize the current state of atomic parameters, highlighting the areas of satisfactory progress and noting places, where further laboratory progress will be welcome.
\end{abstract}

Keywords. atomic data, stars: abundances, stars: Population II

\section{Introduction}

Detailed understanding of the Milky Way's chemical evolution leads to insights on the lives and deaths of element-giving stars, on the return of newly-minted elements to the interstellar medium, on stellar populations, and ultimately on the complex elemental and even isotopic composition of our solar system. Galactic chemical evolution depends on a variety of inputs, but is fundamentally limited by the accuracies of observed abundances of individual stars. Stellar abundance accuracies depend on many factors, such as the quality of observed spectra, the reliability of stellar model atmospheres, the completeness of physical descriptions of atomic and molecular species and energy level populations, and the understanding of radiative transfer in stellar atmospheres. Again there is a fundamental limitation: stellar abundances can be no better than the accuracies of line data (transition probabilities, hyperfine and isotopic substructures) of spectroscopic features that are detectable in stellar spectra.

Here we briefly describe recent laboratory advances in atomic transition data. We concentrate on contributions by the Wisconsin atomic physics group on lines from species of most interest to spectroscopists working on cool stars. Important work has been done by several other groups, in particular those in Lund, Mons, and London, and we comment briefly on their results.

\section{Neutron-Capture Elements}

More than three decades ago came the discovery of low metallicity $([\mathrm{Fe} / \mathrm{H}]<-2) \dagger$ stars with abnormal enhancements of the heavy neutron-capture ( $n$-capture; atomic number

$\dagger$ We adopt the standard spectroscopic notation: for elements $\mathrm{A}$ and $\mathrm{B},[\mathrm{A} / \mathrm{B}] \equiv$ $\log _{10}\left(\mathrm{~N}_{\mathrm{A}} / \mathrm{N}_{\mathrm{B}}\right)_{\star}-\log _{10}\left(\mathrm{~N}_{\mathrm{A}} / \mathrm{N}_{\mathrm{B}}\right)_{\odot}$. We use the definition $\log \epsilon(\mathrm{A}) \equiv \log _{10}\left(\mathrm{~N}_{\mathrm{A}} / \mathrm{N}_{\mathrm{H}}\right)+12.0$, and equate metallicity with the stellar $[\mathrm{Fe} / \mathrm{H}]$ value. 


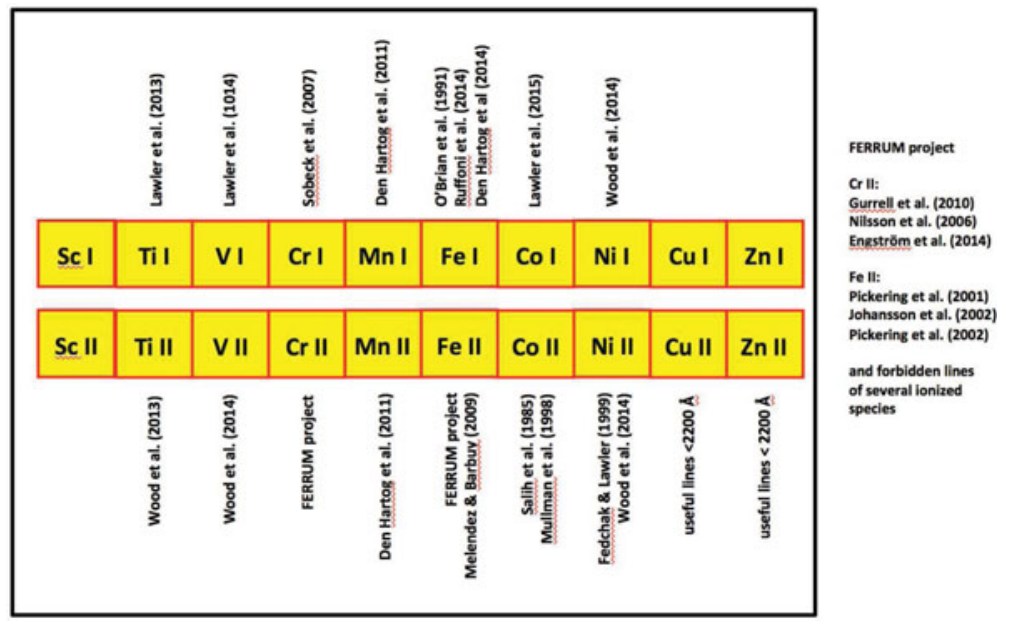

Figure 1. Visual display of neutral and ionized Fe-group species, with labels citing mostly recent extensive laboratory studies. Lack of a reference for a species indicates no recent lab publication, but does not necessarily indicate lack of good older data.

Z > 30) elements (e.g., Griffin et al. 1982). Now many metal-poor stars are known that exhibit enrichments by rapid (the $r$-process) and slow (the $s$-process) $n$-capture synthesis events. A summary of the general properties of $r$ - and $s$-process-rich stars was given in Sneden et al. (2008). For some stars, particularly those with $r$-process enrichments, there are impressive numbers of published $n$-capture elemental abundances. Just to name two examples: CS 31082-001 with 37 elements (Siqueira Mello et al. 2013) and HD 221170 (Ivans et al. 2006) with 35 elements. These stars' $n$-capture abundances show excellent agreement with the scaled solar-system $r$-process-only element distribution. This conclusion is made possible by improvements in observed stellar spectra, and by significant upgrades in the quality and quantity of transition data, especially for the rare-earth elements (summarized in Sneden et al. 2009) and for lighter $n$-capture elements (e.g., Malcheva et al. 2006, Biémont et al. 2011). The excellent agreement between the $n$ capture abundances of $r$-rich metal-poor stars and the solar-system values suggests that the major observational work in this area may have now been accomplished, and further advances mostly may be with theoretical $r$-process predictions. Observers might want to concentrate more on detailed abundance investigations of so-called " $r$-process truncation" stars (Boyd et al. 2012 and references therein) and the $s$-process-rich stars (Placco et al. 2015 and references therein).

\section{Fe-Group Elements}

In contrast to the $n$-capture elements, abundances of Fe-group $(\mathrm{Z}=21-30, \mathrm{Sc}-\mathrm{Zn})$ elements have not reached a level of accuracy to really constrain stellar nucleosynthesis models. This is disappointing because theoretical models can predict the Fe-group elemental abundances in some detail (e.g., Kobayashi et al. 2011 and references therein).

The Fe-group observed abundance reliability has been limited by (1) lack of transitions of both neutral and ionized species of several elements in the usually studied optical spectral region $(\lambda>4000 \AA)$; (2) concerns about large departures from local thermodynamic equilibrium in lines of especially the neutral species; (3) and most importantly, less-thandesired quality in laboratory $g f$ values and hyperfine substructures. Fortunately, several groups are making much progress in lab spectroscopy of these elements. We depict the 


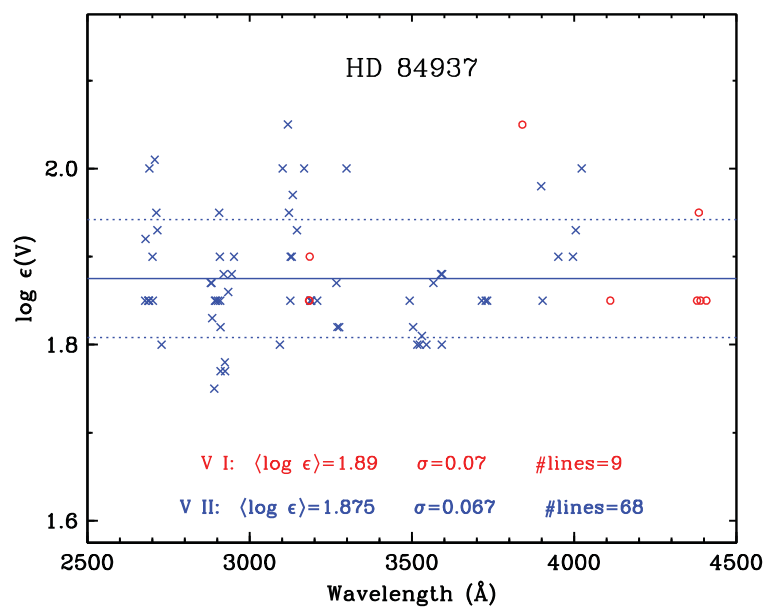

Figure 2. Vanadium abundances in the warm main-sequence very metal-poor star HD 84937, from V I and V II transitions. This figure is taken from Lawler et al. (2014). The abundance statistics are written in the figure legend.

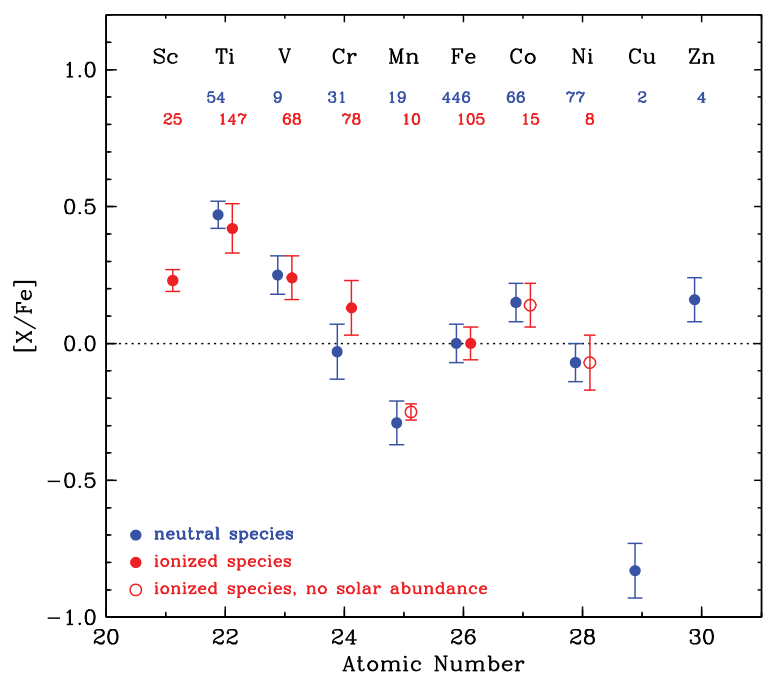

Figure 3. Abundance ratios of Fe-group elements with respect to Fe in the warm main-sequence very metal-poor star HD 84937. The symbol meanings are given in the figure legend. The number of lines that have contributed to each species abundance is written beneath its element name.

current state of published Fe-group transition papers in Fig. 1. By inspection one can easily see that most Fe-group species have received renewed lab scrutiny in the last decade.

As an example of application of new lab data to the stellar spectra, Fig. 2, taken from Lawler et al. (2014), shows abundances from individual V I and V II lines in the spectrum of the very metal-poor $([\mathrm{Fe} / \mathrm{H}] \simeq-2.3)$ warm $\left(T_{\text {eff }} \simeq 6250 \mathrm{~K}\right)$ main sequence star HD 84937. Several conclusions can be drawn here. (1) Mean abundances from the two species are in agreement, and the line-line abundance scatters are relatively small. (2) Only a few V I lines are detectable; V is mostly ionized, and the metallicity of HD 84937 is low. Both effects conspire to weaken V I transitions. (3) A rich spectrum of V II can be 
seen, but only by obtaining spectra in the near- $U V(3000-4000 \AA)$ and the vacuum- $U V$ (2300-3000 ̊).

We have recently completed a comprehensive Fe-group abundance study of HD 84937 (Sneden et al. 2015). A summary of our results is shown in Fig. 3. It is clear that neutral and ionized species yield the same elemental abundances for seven of the Fe-group elements; Saha ionization balance holds in this stellar atmosphere. More interesting astrophysically, there are pronounced departures from the solar chemical composition. This is especially seen in the (unexpected) overabundances of the three lightest elements Sc, $\mathrm{Ti}$, and V. This is not predicted in standard massive-star nucleosynthesis models. Further discussion of these abundance anomalies are given in Sneden et al. A general (but detailed) Fe-group abundance survey in other low metallicity stars should be undertaken.

This work has been supported in part by NASA grant NNX10AN93G (J.E.L.), by NSF grant AST-1211055 (J.E.L.) and NSF grant AST-1211585 (C.S.).

\section{References}

Biémont, É., Blagoev, K., Engström, L., et al. 2011, MNRAS, 414, 3350

Boyd, R. N., Famiano, M. A., Meyer, B. S., et al. 2012, ApJL, 744, L14

Den Hartog, E. A., Ruffoni, M. P., Lawler, J. E., et al. 2014, ApJS, 215, 23

Den Hartog, E. A., Lawler, J. E., Sobeck, J. S., Sneden, C., \& Cowan, J. J. 2011, ApJS, 194, 35

Engström, L., Lundberg, H., Nilsson, H., Hartman, H., \& Bäckström, E. 2014, A\&SA, 570, A34

Fedchak, J. A. \& Lawler, J. E. 1999, ApJ, 523, 734

Griffin, R., Gustafsson, B., Vieira, T., \& Griffin, R. 1982, MNRAS, 198, 637

Gurell, J., Nilsson, H., Engström, L., et al. 2010, A\&A, 511, A68

Ivans, I. I., Simmerer, J., Sneden, C., et al. 2006, ApJ, 645, 613

Johansson, S., Derkatch, A., Donnelly, M. P., et al. 2002, Phys. Scr. T, 100, 71

Kobayashi, C., Karakas, A. I., \& Umeda, H. 2011, MNRAS, 414, 3231

Lawler, J. E., Guzman, A., Wood, M. P., Sneden, C., \& Cowan, J. J. 2013, ApJS, 205, 11

Lawler, J. E., Sneden, C., \& Cowan, J. J. 2015, ApJS, 220, 13

Lawler, J. E., Wood, M. P., Den Hartog, E. A., et al. 2014, ApJS, 215, 20

Malcheva, G., Blagoev, K., Mayo, R., et al. 2006, MNRAS, 367, 754

Meléndez, J. \& Barbuy, B. 2009, A\&A, 497, 611

Mullman, K. L., Cooper, J. C., \& Lawler, J. E. 1998, ApJ, 495, 503

Nilsson, H., Ljung, G., Lundberg, H., \& Nielsen, K. E. 2006, A\&A, 445, 1165

O'Brian, T. R., Wickliffe, M. E., Lawler, J. E., Whaling, W., \& Brault, J. W. 1991, Journal of the Optical Society of America B Optical Physics, 8, 1185

Pickering, J. C., Johansson, S., \& Smith, P. L. 2001, A\& A, 377, 361

Pickering, J. C., Donnelly, M. P., Nilsson, H., Hibbert, A., \& Johansson, S. 2002, A\& A, 396, 715

Placco, V. M., Beers, T. C., Ivans, I. I., et al. 2015, ApJ, 812, 109

Ruffoni, M. P., Den Hartog, E. A., Lawler, J. E., et al. 2014, MNRAS, 441, 3127

Siqueira Mello, C., Spite, M., Barbuy, B., et al. 2013, A\&\&A, 550, A122

Sneden, C., Cowan, J. J., \& Gallino, R. 2008, ARA\&A, 46, 241

Sneden, C., Cowan, J. J., Pignatari, M., Kobayashi, C., Lawler, J. E., Den Hartog, E. A., \& Wood, M. P. 2015, ApJS, submitted

Sneden, C., Lawler, J. E., Cowan, J. J., Ivans, I. I., \& Den Hartog, E. A. 2009, ApJS, 182, 80

Sobeck, J. S., Lawler, J. E., \& Sneden, C. 2007, ApJ, 667, 1267

Wood, M. P., Lawler, J. E., Den Hartog, E. A., Sneden, C., \& Cowan, J. J. 2014a, ApJS, 214, 18

Wood, M. P., Lawler, J. E., Sneden, C., \& Cowan, J. J. 2013, ApJS, 208, 27

Wood, M. P., Lawler, J. E., Sneden, C., \& Cowan, J. J. 2014b, ApJS, 211, 20 